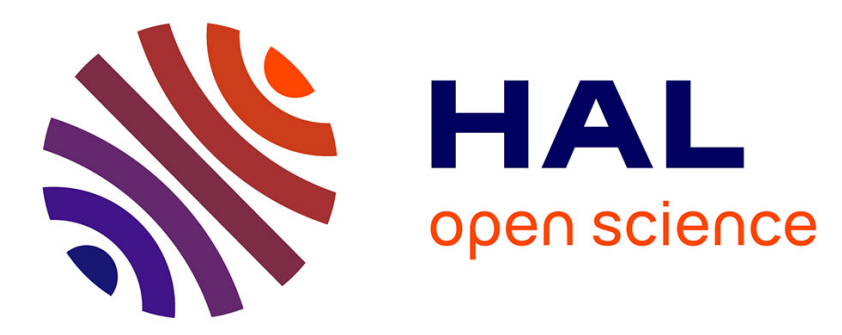

\title{
Mammographic evidence of microenvironment changes in tumorous breasts
}

Zach Marin, Kendra A. Batchelder, Brian C. Toner, Lyne Guimond, Evgeniya G Gerasimova-Chechkina, Amy R. Harrow, Alain Arneodo, Andre Khalil

\section{- To cite this version:}

Zach Marin, Kendra A. Batchelder, Brian C. Toner, Lyne Guimond, Evgeniya G GerasimovaChechkina, et al.. Mammographic evidence of microenvironment changes in tumorous breasts. Medical Physics, 2017, 44 (4), pp.1324-1336. 10.1002/mp.12120 . hal-01541567

\section{HAL Id: hal-01541567 \\ https://hal.science/hal-01541567}

Submitted on 19 Jun 2017

HAL is a multi-disciplinary open access archive for the deposit and dissemination of scientific research documents, whether they are published or not. The documents may come from teaching and research institutions in France or abroad, or from public or private research centers.
L'archive ouverte pluridisciplinaire HAL, est destinée au dépôt et à la diffusion de documents scientifiques de niveau recherche, publiés ou non, émanant des établissements d'enseignement et de recherche français ou étrangers, des laboratoires publics ou privés.

\section{다(1)(2)}

Distributed under a Creative Commons Attribution - ShareAlikel 4.0 International 


\title{
Mammographic evidence of microenvironment changes in tumorous breasts
}

\author{
Zach Marin, Kendra A. Batchelder, Brian C. Toner, and Lyne Guimond \\ CompuMAINE Laboratory, Department of Mathematics \& Statistics, University of Maine, Orono, ME 04469, USA \\ Evgeniya Gerasimova-Chechkina \\ Laboratory of Physical Foundation of Strength, Institute of Continuous Media Mechanics UB RAS, Perm, Russia
}

Amy R. Harrow

Spectrum Medical Group, Eastern Maine Medical Center, Bangor, ME 04401, USA

Alain Arneodo
LOMA, Universite de Bordeaux, CNRS, UMR 5798, 33405 Talence, France

Andre Khalil ${ }^{\text {a) }}$

CompuMAINE Laboratory, Department of Mathematics \& Statistics, University of Maine, Orono, ME 04469, USA

Purpose: The microenvironment of breast tumors plays a critical role in tumorigenesis. As long as the structural integrity of the microenvironment is upheld, the tumor is suppressed. If tissue structure is lost through disruptions in the normal cell cycle, the microenvironment may act as a tumor promoter. Therefore, the properties that distinguish between healthy and tumorous tissues may not be solely in the tumor characteristics but rather in surrounding non-tumor tissue. The goal of this paper was to show preliminary evidence that tissue disruption and loss of homeostasis in breast tissue microenvironment and breast bilateral asymmetry can be quantitatively and objectively assessed from mammography via a localized, wavelet-based analysis of the whole breast.

Methods: A wavelet-based multifractal formalism called the 2D Wavelet Transform Modulus Maxima (WTMM) method was used to quantitate density fluctuations from mammographic breast tissue via the Hurst exponent $(H)$. Each entire mammogram was cut in hundreds of $360 \times 360$ pixel subregions in a gridding scheme of overlapping sliding windows, with each window boundary separated by 32 pixels. The 2D WTMM method was applied to each subregion individually. A data mining approach was set up to determine which metrics best discriminated between normal vs. cancer cases. These same metrics were then used, without modification, to discriminate between normal vs. benign and benign vs. cancer cases.

Results: The density fluctuations in healthy mammographic breast tissue are either monofractal anti-correlated $(H<1 / 2)$ for fatty tissue or monofractal long-range correlated $(H>1 / 2)$ for dense tissue. However, tissue regions with $H \sim 1 / 2$, as well as left vs. right breast asymetries, were found preferably in tumorous (benign or cancer) breasts vs. normal breasts, as quantified via a combination metric yielding a $P$-value $\sim 0.0006$. No metric considered showed significant differences between cancer vs. benign breasts.

Conclusions: Since mammographic tissue regions associated with uncorrelated $(H \sim 1 / 2)$ density fluctuations were predominantly in tumorous breasts, and since the underlying physical processes associ-

ated with a $H \sim 1 / 2$ signature are those of randomness, lack of spatial correlation, and free diffusion, it is hypothesized that this signature is also associated with tissue disruption and loss of tissue homeostasis.

Key words: Hurst exponent, radiomics, tissue disruption, tissue homeostasis, wavelets

\section{Abbreviations}

CC craniocaudal

DDSM digital database for screening mammography

FFDM full field digital mammography

MLO mediolateral oblique

WTMMM

wavelet transform modulus maxima maxima

WTMM wavelet transform modulus maxima

WT wavelet transform

TME tumor microenvironment

\section{INTRODUCTION}

This paper examines the hypothesis that breast tissue disruption and loss of tissue homeostasis in the microenvironment of malignant breast tumors is detectable via a quantitative and objective roughness analysis of whole breast mammograms.

Findings suggest that differences between normal and cancerous cells and their interactions exist across multiple size scales. Indeed, when functioning properly, the cellular 
microenvironment impedes cancerous cell growth, which leads to death of the cancerous cells or quiescence of an occult tumor. ${ }^{1}$ When functioning poorly, the environment of cancerous lesions creates a niche favoring the survival of cancerous stem cells and protecting cells from treatment or therapy. ${ }^{2}$ Alteration of the stromal architecture and composition of the extracellular matrix is a well-recognized component of both benign and malignant breast pathologies. ${ }^{1,3}$ Histologically normal breast tissues adjacent to breast tumors frequently exhibit methylation changes in multiple genes. ${ }^{4}$ Highly methylated tumor suppressor genes have been found in peritumoral breast tissue cells as far as $4 \mathrm{~cm}$ away from tumors. ${ }^{5}$ The multicellular architecture and organization of the ductal tree of the mammary gland exhibit coherent cellular movement within an extracellular matrix cocoon, which guides formation of larger structural units of tissues important for quiescence and homeostasis. A disruption of coherent angular motion and the consequential adoption of randomized cellular motility are associated with malignant growth in breast tissue. $^{6}$

Many image analysis techniques were developed to detect and/or assess masses and calcifications in mammograms. ${ }^{7-10}$ But, far fewer studies investigated the structure and arrangement of the non-tumor tissue that contributes to bilateral asymmetry and architectural distortion of breast tissue. ${ }^{11}$ Computational researchers explored breast tissue patterns on mammograms, but most analyzed only the central part of the image and/or failed to connect the texture analysis to known biological tumor processes related to the onset and development of cancer. ${ }^{12-15}$ Some used power spectral analysis to seek correlations between $\beta$ (the power-law exponent of the power spectrum), obtained from a region of interest behind the nipple, and genetic factors. ${ }^{16}$ These authors concluded that BRCA1/2 gene mutation carriers and low-risk women had different mammographic parenchymal patterns. Researchers also showed that BRCA1/2 mutation carriers could be identified via computer-extracted mammographic texture patterns, but not by mammographic density analysis. $^{17}$

Power spectral analysis was also used to show that the power spectrum exponent of mammograms is approximately $\beta=3 \cdot{ }^{18}$ Note that when averaging over many noisy processes and many different mammograms, and/or different subregions within a mammogram, one can expect subregions where $\beta \sim 2.5-2.8$ and those where $\beta \sim 3.2-3.5$ to average and yield $\beta \sim 3$. Since this is a possibility, analyses should refrain from averaging processes when computing the roughness of mammograms, and instead obtain sets of subregionspecific $\beta$ values. In addition, the spectral analysis yielding $\beta$ is intrinsically unable to discriminate between monofractal signatures (where the roughness is homogeneously distributed - and quantified via a single exponent such as $\beta$ ) vs. multifractal signatures (where the roughness is defined locally - and quantified via a whole spectrum of roughness exponents). ${ }^{10,19-30}$ This inability to discriminate between monofractal vs. multifractal signatures is absolutely critical in this context. It can lead to unknowingly averaging two different monofractal signatures within the same image (e.g., a subregion contains anti-correlated structures and another subregion with long-range correlations) to yield a false global uncorrelated signature.

The Wavelet Transform Modulus Maxima (WTMM) method is a multifractal formalism used to analyze complex 1D signals, ${ }^{19,20,29,30}$ 2D images, ${ }^{10,23-28,31-36} 3 \mathrm{D}$ images, ${ }^{37}$ and vector fields. ${ }^{38}$ By considering a partition function obtained from the so-called wavelet-transform space-scale skeleton, and by exploring several different statistical order moments, one can extract a singularity spectrum yielding quantitative information on the monofractal vs. multifractal nature of a given image.

Fifteen years ago, in an exploratory analysis of digitized mammograms using the 2D WTMM method, Kestener et al. ${ }^{26}$ introduced the possibility of using the Hurst exponent, $H$, which quantifies the global roughness of the image density fluctuations, ${ }^{\dagger}$ as a potential tool to discriminate between dense and fatty breast tissue. High mammographic breast tissue density is a risk factor for breast cancer, by a $\sim 5$ fold factor compared to low density. ${ }^{39}$ Kestener et al.'s results showed that normal regions display monofractal scaling properties as characterized by $H \sim 0.30$ in fatty areas, which look like anti-persistent self-similar random surfaces, vs. $H \sim 0.65$ in dense areas, which exhibit long-range correlations. Indeed, for $0<H<1 / 2$, density fluctuations are spatially anti-correlated. For $1 / 2<H<1$, the fluctuations are spatially positively correlated. Interestingly, for $H \sim 1 / 2$, the fluctuations are uncorrelated and are associated with random physical processes like Brownian motion and free diffusion.

A high percentage of breast cancers go undetected, as evidenced by surveys of autopsy reports. Undiagnosed occult breast cancers have been found in women who died from unrelated causes at rates as high as $15.6 \%$ according to one study ${ }^{40}$ and $19 \%$ according to another, which also found this rate as high as $39 \%$ for women in their forties. ${ }^{41}$ Disrupted tissue density fluctuations may be indicative of these occult tumors, and detection of these fluctuations may pave the way for further research into finding cancer at an earlier stage.

To study the architectural landscape of breast tissue density fluctuations, the 2D WTMM method ${ }^{10,23-28,31-36}$ was used to analyze entire breast mammograms from normal, cancer, and benign cases taken from the Digital Database for Screening Mammography (DDSM) ${ }^{42,43}$ Only microcalcification cases were analyzed for cancer and benign. The analysis was performed on 43 normal, 49 cancer, and 35 benign cases. Following the calculation of the monofractal scaling exponent $H$ at a fine level, using a sliding window analysis for each mammogram studied, a data mining approach was implemented to determine which parameters are most effective at distinguishing between normal and cancer cases. The benign cases were then compared to both the normal cases and cancer cases using these calibrated parameters. The results demonstrate that disrupted regions associated with

\footnotetext{
${ }^{\dagger}$ The relationship between $H$ and $\beta$ is given by $H \quad(\beta 2) / 2$ for monofractal rough surfaces.
} 
loss of tissue homeostasis, as quantified by $H \sim 1 / 2$ and loss of breast symmetry, are found significantly in tumorous cases (cancer or benign) when compared to normal cases $(P \sim$ $0.0006)$. Significant differences were also found individually between cancer vs. normal cases $(P \sim 0.0023)$ and benign vs. normal cases $(P \sim 0.0049)$, while no significant differences were found between benign vs. cancer cases $(P \sim 0.85)$.

\section{METHODS}

\section{A. The data}

Mammograms were obtained from the digital database for screening mammography (DDSM) at the University of South Florida. ${ }^{42,43}$ The databank contains over 2600 studies made up of normal, benign, benign without call back, and cancer mammograms, all categorized by expert radiologists. Each study had two images of each breast: a mediolateral oblique (MLO) view and a craniocaudal (CC) view. Non-normal DDSM cases may have benign or malignant masses and/or microcalcifications, but only cancer or benign cases containing exactly one tumor were looked at in this study. Only the MLO view was analyzed. The lesion itself was not the centerpiece of this research. Instead, the study examined a lesion's influence on the whole breast. Nevertheless, in case masses vs. microcalcifications differ in their effect on microenvironments, all non-normal cases considered in this study were microcalcification cases.

A total of 127 cases were considered, corresponding to 254 entire breast mammogram images: 43 normal cases (86 images), 49 cancerous cases (98 images), and 35 benign cases (70 images). Tables I, II, III contain descriptive information about all cases considered. Breast image masks were created in ImageJ by manually encircling the breast, thus ensuring that extraneous background tissue (i.e., the pectoral muscle) did not influence the analysis.

\section{B. The 2D WTMM method}

The strategy behind the 2D Wavelet Transform Modulus Maxima (WTMM) method ${ }^{10,23,26-28,31-33,35,36,44-48}$ is to use the continuous wavelet transform (WT) as a mathematical microscope to characterize image density fluctuations over a continuous range of size scales. The WT of an image is the gradient vector of the image smoothed by dilated versions of a Gaussian filter. At each size scale, the WTMM are defined by the positions where the modulus of the WT is locally maximal. These WTMM are organized as maxima chains, parameterized by position, at the considered scale. The maxima chains for three different size scales are shown in black in Figs 1(b1-3), 1(c1-3), 1(d1-3). Along each of these chains, further local maxima are found, i.e., the WTMM maxima (WTMMM), shown as blue, red, and yellow arrows, respectively in Figs 1(b1-3), 1(c1-3), 1(d1-3). The WTMMM from each scale are then linked to define the maxima lines that form the WT skeleton (Figs 1(b4), 1(c4), 1(d4)). By considering how the WT modulus varies with scale along each
TABLE I. Normal cases.

\begin{tabular}{|c|c|c|c|c|c|c|}
\hline Type & Folder & Case & Scanner & Resolution & Age & Density \\
\hline Normal & Normal 04 & Case 0412 & DBA & 42 & 49 & 2 \\
\hline Normal & Normal 04 & Case0415 & DBA & 42 & 41 & 1 \\
\hline Normal & Normal 04 & Case0416 & DBA & 42 & 53 & 3 \\
\hline Normal & Normal 04 & Case0417 & DBA & 42 & 78 & 2 \\
\hline Normal & Normal 04 & Case0418 & DBA & 42 & 43 & 4 \\
\hline Normal & Normal 04 & Case0419 & DBA & 42 & 68 & 2 \\
\hline Normal & Normal 04 & Case0420 & DBA & 42 & 51 & 1 \\
\hline Normal & Normal 04 & Case0422 & DBA & 42 & 57 & 1 \\
\hline Normal & Normal 04 & Case 0423 & DBA & 42 & 63 & 2 \\
\hline Normal & Normal 04 & Case0424 & DBA & 42 & 48 & 3 \\
\hline Normal & Normal 04 & Case0425 & DBA & 42 & 61 & 2 \\
\hline Normal & Normal 04 & Case0426 & DBA & 42 & 50 & 4 \\
\hline Normal & Normal 04 & Case0427 & DBA & 42 & 52 & 2 \\
\hline Normal & Normal 04 & Case0431 & DBA & 42 & 71 & 2 \\
\hline Normal & Normal 04 & Case0432 & DBA & 42 & 40 & 3 \\
\hline Normal & Normal 04 & Case0433 & DBA & 42 & 41 & 3 \\
\hline Normal & Normal 04 & Case0434 & DBA & 42 & 65 & 4 \\
\hline Normal & Normal 04 & Case0435 & DBA & 42 & 45 & 4 \\
\hline Normal & Normal 04 & Case0436 & DBA & 42 & 66 & 3 \\
\hline Normal & Normal 04 & Case0437 & DBA & 42 & 42 & 4 \\
\hline Normal & Normal 04 & Case0438 & DBA & 42 & 70 & 2 \\
\hline Normal & Normal 04 & Case0439 & DBA & 42 & 71 & 3 \\
\hline Normal & Normal 04 & Case 0440 & DBA & 42 & 47 & 4 \\
\hline Normal & Normal 04 & Case0441 & DBA & 42 & 40 & 3 \\
\hline Normal & Normal 04 & Case0442 & DBA & 42 & 80 & 2 \\
\hline Normal & Normal 04 & Case0443 & DBA & 42 & 78 & 3 \\
\hline Normal & Normal 04 & Case0444 & DBA & 42 & 68 & 3 \\
\hline Normal & Normal 04 & Case0445 & DBA & 42 & 75 & 2 \\
\hline Normal & Normal 04 & Case0447 & DBA & 42 & 42 & 1 \\
\hline Normal & Normal 04 & Case0448 & DBA & 42 & 51 & 4 \\
\hline Normal & Normal 04 & Case0449 & DBA & 42 & 60 & 2 \\
\hline Normal & Normal 04 & Case0450 & DBA & 42 & 64 & 2 \\
\hline Normal & Normal 04 & Case0462 & DBA & 42 & 62 & 2 \\
\hline Normal & Normal 04 & Case 0463 & DBA & 42 & 66 & 3 \\
\hline Normal & Normal 04 & Case0464 & DBA & 42 & 71 & 2 \\
\hline Normal & Normal 04 & Case0465 & DBA & 42 & 56 & 1 \\
\hline Normal & Normal 04 & Case0466 & DBA & 42 & 68 & 3 \\
\hline Normal & Normal 04 & Case0467 & DBA & 42 & 70 & 4 \\
\hline Normal & Normal 04 & Case0468 & DBA & 42 & 62 & 2 \\
\hline Normal & Normal 04 & Case0469 & DBA & 42 & 64 & 3 \\
\hline Normal & Normal 04 & Case 0470 & DBA & 42 & 51 & 4 \\
\hline Normal & Normal 04 & Case0471 & DBA & 42 & 71 & 2 \\
\hline Normal & Normal 04 & Case0472 & DBA & 42 & 70 & 1 \\
\hline Normal & Normal 04 & Case0475 & DBA & 42 & 57 & 3 \\
\hline Normal & Normal 04 & Case0477 & DBA & 42 & 56 & 1 \\
\hline Normal & Normal 04 & Case0478 & DBA & 42 & 67 & 1 \\
\hline \multirow[t]{3}{*}{ Normal } & Normal 04 & Case0479 & DBA & 42 & 61 & 2 \\
\hline & & & & Average & 59.2 & 2.5 \\
\hline & & & & Stdev & 11.5 & 1.0 \\
\hline
\end{tabular}

maxima line, one can obtain the so-called singularity spectrum $^{23-25}$ for each subset (see below), which allows quantitative discrimination between monofractal vs. multifractal 
TABle II. Cancer cases.

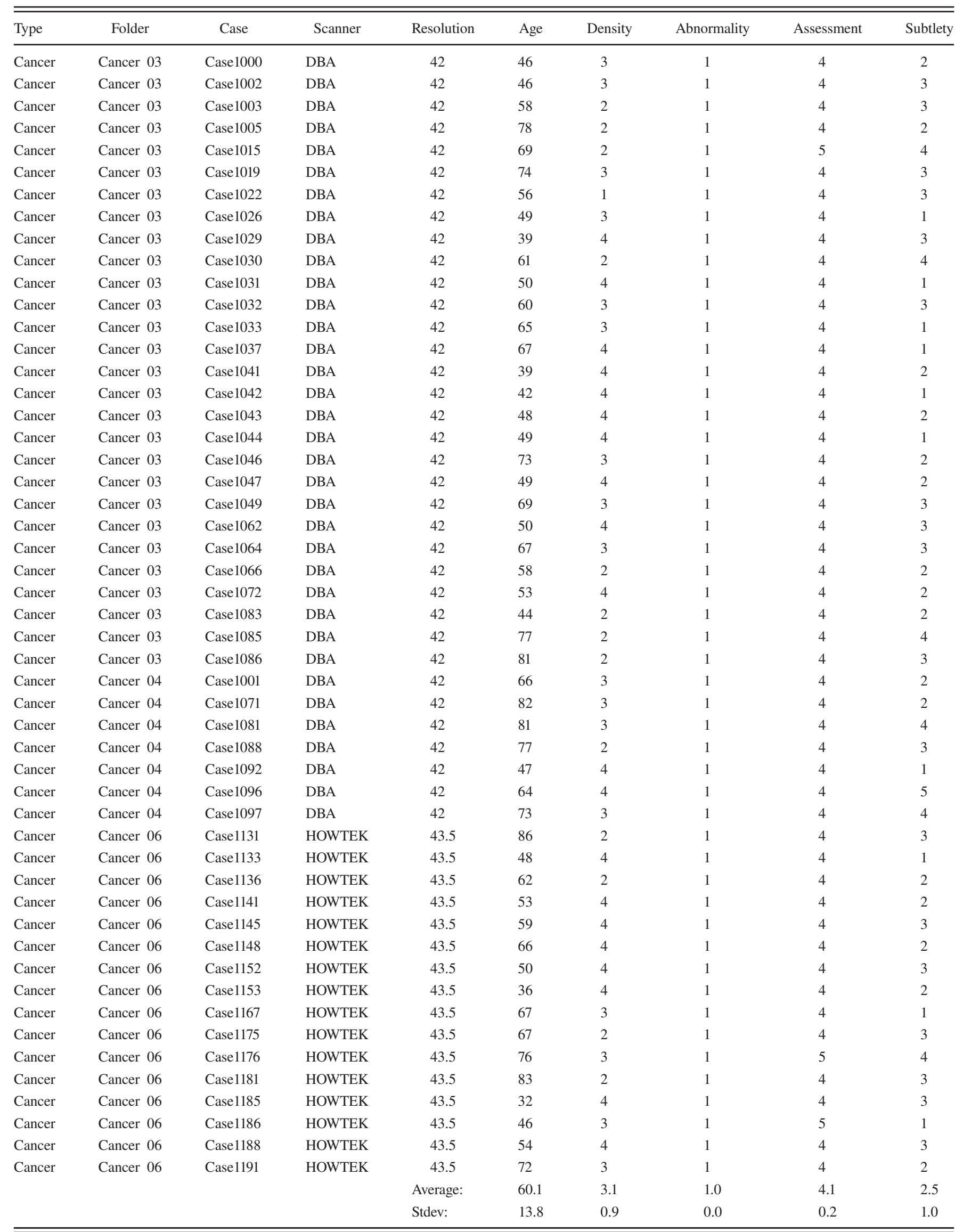


TABLE III. Benign cases.

\begin{tabular}{|c|c|c|c|c|c|c|c|c|c|}
\hline Benign & Benign 02 & Case1123 & HOWTEK & 43.5 & 56 & 3 & 1 & 4 & 4 \\
\hline Benign & Benign 02 & Case1242 & HOWTEK & 43.5 & 79 & 3 & 1 & 4 & 2 \\
\hline Benign & Benign 02 & Case1265 & HOWTEK & 43.5 & 48 & 3 & 1 & 4 & 3 \\
\hline Benign & Benign 02 & Case1269 & HOWTEK & 43.5 & 44 & 3 & 1 & 4 & 3 \\
\hline Benign & Benign 02 & Case 1270 & HOWTEK & 43.5 & 54 & 4 & 1 & 4 & 4 \\
\hline Benign & Benign 02 & Case1276 & HOWTEK & 43.5 & 80 & 3 & 1 & 4 & 4 \\
\hline Benign & Benign 02 & Case1278 & HOWTEK & 43.5 & 50 & 2 & 1 & 4 & 2 \\
\hline Benign & Benign 02 & Case1280 & HOWTEK & 43.5 & 50 & 4 & 1 & 4 & 2 \\
\hline Benign & Benign 02 & Case 1285 & HOWTEK & 43.5 & 63 & 4 & 1 & 4 & 4 \\
\hline Benign & Benign 02 & Case 1300 & HOWTEK & 43.5 & 52 & 3 & 1 & 4 & 4 \\
\hline Benign & Benign 02 & Case1324 & HOWTEK & 43.5 & 50 & 4 & 1 & 4 & 3 \\
\hline Benign & Benign 02 & Case1326 & HOWTEK & 43.5 & 44 & 4 & 1 & 4 & 4 \\
\hline Benign & Benign 02 & Case1327 & HOWTEK & 43.5 & 58 & 4 & 1 & 4 & 4 \\
\hline Benign & Benign 02 & Case1328 & HOWTEK & 43.5 & 62 & 4 & 1 & 4 & 5 \\
\hline Benign & Benign 02 & Case 1330 & HOWTEK & 43.5 & 64 & 3 & 1 & 4 & 2 \\
\hline Benign & Benign 02 & Case1331 & HOWTEK & 43.5 & 73 & 1 & 1 & 4 & 1 \\
\hline Benign & Benign 02 & Case1333 & HOWTEK & 43.5 & 58 & 1 & 1 & 4 & 2 \\
\hline Benign & Benign 03 & Case1259 & HOWTEK & 43.5 & 48 & 4 & 1 & 4 & 2 \\
\hline Benign & Benign 03 & Case1332 & HOWTEK & 43.5 & 65 & 2 & 1 & 4 & 2 \\
\hline Benign & Benign 03 & Case1364 & HOWTEK & 43.5 & 42 & 4 & 1 & 4 & 3 \\
\hline Benign & Benign 03 & Case1406 & HOWTEK & 43.5 & 75 & 1 & 1 & 4 & 4 \\
\hline Benign & Benign 03 & Case1427 & HOWTEK & 43.5 & 59 & 3 & 1 & 4 & 3 \\
\hline \multirow[t]{3}{*}{ Benign } & Benign 03 & Case1433 & HOWTEK & 43.5 & 58 & 3 & 1 & 2 & 3 \\
\hline & & & & Average: & 58.6 & 3.0 & 1.0 & 3.9 & 3.0 \\
\hline & & & & Stdev: & 11.0 & 1.0 & 0.0 & 0.4 & 1.3 \\
\hline
\end{tabular}

processes. In the monofractal case, the tissue in the tumor microenvironment is characterized by the roughness exponent $H$ one subregion at a time. A technical presentation follows.

Each subregion of the mammographic image can be viewed as a single-valued, self-affine function, $f(\mathbf{x}){ }^{26}$ The WT is defined as (in vectorial form)

$$
\mathbf{T}_{\psi}[f](\mathbf{b}, a)=\left(\begin{array}{c}
T_{\psi_{1}}[f]=a^{2} \int d^{2} \mathbf{x} \psi_{1}\left(a^{1}(\mathbf{x}-\mathbf{b})\right) f(\mathbf{x}) \\
T_{\psi 2}[f]=a^{2} \int d^{2} \mathbf{x} \psi_{2}\left(a^{1}(\mathbf{x}-\mathbf{b})\right) f(\mathbf{x})
\end{array}\right),
$$

where $\psi_{1}(x, y)=\frac{\partial \phi(x, y)}{\partial x}, \psi_{2}(x, y)=\frac{\partial \phi(x, y)}{\partial y}$, and $\phi(x, y)$ is a $2 \mathrm{D}$ Gaussian isotropic function. The WT can be expressed in terms of the modulus and argument

$$
\mathcal{M}_{\psi}[f](\mathbf{b}, a)=\left[\left(T_{\psi_{1}}[f](\mathbf{b}, a)\right)^{2}+\left(T_{\psi_{2}}[f](\mathbf{b}, a)\right)^{2}\right]^{1 / 2},
$$

$$
\mathcal{A}_{\psi}[f](\mathbf{b}, a)=\operatorname{Arg}\left(T_{\psi_{1}}[f](\mathbf{b}, a)+i T_{\psi_{2}}[f](\mathbf{b}, a)\right),
$$

The WTMM are defined as the positions where the modulus is locally maximum in the direction of the argument. These WTMM form connected chains called maxima chains. The WTMMM are defined as the local maxima along the maxima chains. The wavelet transform space-scale skeleton can be calculated by linking all WTMMM across all scales $a>0$. Then let $\mathcal{L}(a)$ be the set of maxima lines that exist at any scale $a$, and define the partition functions 

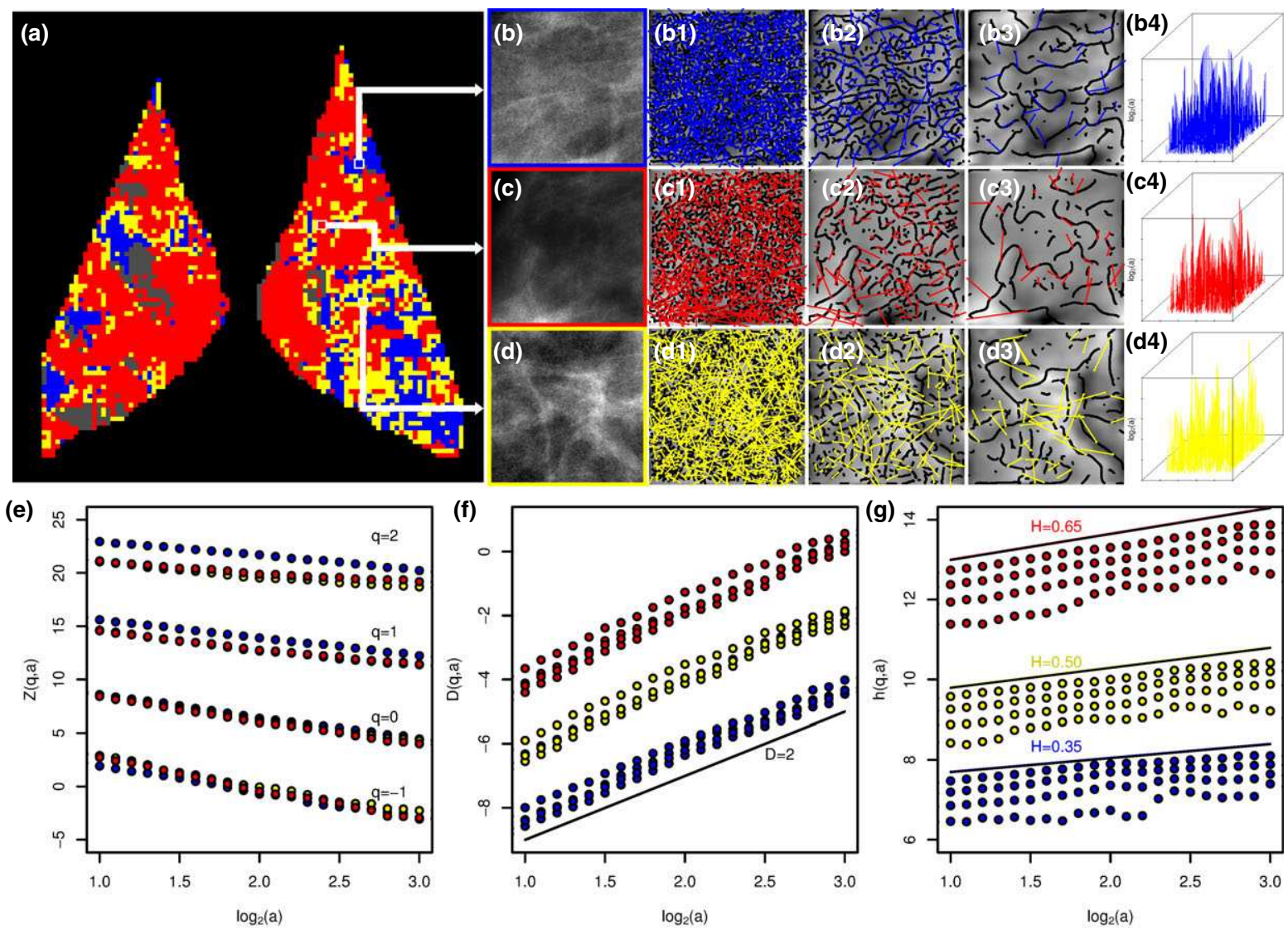

(h)

(i)

(j)
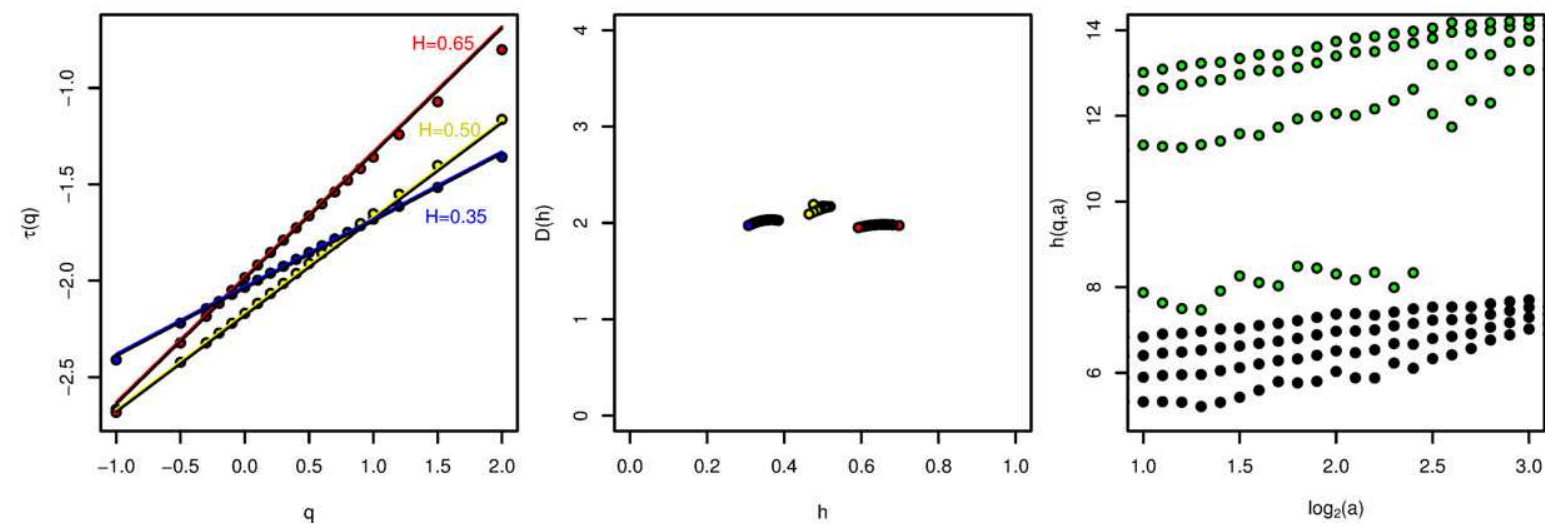

Fig. 1. Sliding window WTMM analysis of DDSM cancer 03, case 1022. (a) Color coded MLO views of the right breast (shown on left) and the cancerous left breast (shown on right). Each pixel represents a $360 \times 360$ pixel mammogram subregion colored according to its $H$ value. Subregions where $H \leq 0.45$ (fatty) are colored blue, $0.45<H<0.55$ (disrupted) are yellow, and $H \geq 0.55$ (dense) are red. Gray pixels correspond to rejected subregions (see text). [(b) (d), 13 ] WTMM maxima chains (black) and WTMMM (colored, with arrows representing the WT vector) at small (b1), (c1), (d1), medium (b2), (c2), (d2), and large (b3), (c3), (d3) scales. [(b) (d), 4] WTMM skeletons formed from chaining WTMMM across every scale. (e) (g) Plots of $\boldsymbol{Z}(\boldsymbol{q}, \boldsymbol{a})$ [Eq. (3)], $\boldsymbol{D}(\boldsymbol{q}, \boldsymbol{a})$ [Eq. (8)], and $\boldsymbol{h}(\boldsymbol{q}, \boldsymbol{a})$ [Eq. (7)] vs. $\log _{2}(\boldsymbol{a})$, respectively, for values of q from 1 (bottom) to 2 (top), from a range of scales of $\log _{2}(\boldsymbol{a}) \quad 1.0$ to 3.0 (in $\boldsymbol{\sigma}_{\boldsymbol{w}}$ units, where $\boldsymbol{\sigma}_{\boldsymbol{w}} 7$ pixels). (H) Plot of $\tau(\boldsymbol{q})$ [Eq. (4)] displaying the monofractal nature of selected subregions and estimations of $H$. (i) Plot of $\boldsymbol{D}(\boldsymbol{h})$ [Eqs. (9), (10)] showing the monofractal nature of selected subregions and estimations of $D$. (j) $\boldsymbol{h}(\boldsymbol{q}, \boldsymbol{a})$ vs. $\log _{2}(\boldsymbol{a})$ for two example subregions rejected due to multifractality (black) and bad scaling (green).

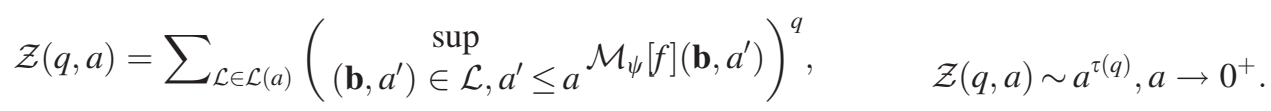

where $q$ are statistical order moments. From the power-law behavior of the partition function one can define the $\tau(q)$ exponents
The $D(h)$ singularity spectrum of $f$ can be determined from the Legendre transform of the partition function scaling exponent 


$$
D(h)=\min _{q}(q h-\tau(q)) .
$$

Due to computational instabilities when using the Legendre transform, one can alternatively use $h$ and $D(h)$ as mean quantities defined in a canonical ensemble, i.e., with respect to their Boltzmann weights computed from the WTMMM

$$
W_{\psi}[f](q, \mathcal{L}, a)=\frac{\left|\sup _{\left(\mathbf{b}, a^{\prime}\right) \in \mathcal{L}, a^{\prime} \leq a} \mathcal{M}_{\psi}[f]\left(\mathbf{b}, a^{\prime}\right)\right|^{q}}{\mathcal{Z}(q, a)} .
$$

Then the expectations can be computed by:

$$
h(q, a)=\sum_{\mathcal{L} \in \mathcal{L}(a)} \ln \mid \begin{aligned}
& \sup _{\left(\mathbf{b}, a^{\prime}\right) \in \mathcal{L}, a^{\prime} \leq a} \mathcal{M}_{\psi}[f]\left(\mathbf{b}, a^{\prime}\right) \mid \\
& W_{\psi}[f](q, \mathcal{L}, a),
\end{aligned}
$$

and

$$
D(q, a)=\sum_{\mathcal{L} \in \mathcal{L}(a)} W_{\psi}[f](q, \mathcal{L}, a) \ln \left(W_{\psi}[f](q, \mathcal{L}, a)\right),
$$

and so

$$
h(q)=\frac{d \tau(q)}{d q}=\lim _{a \rightarrow 0^{+}} h(q, a) / \ln a,
$$

and

$$
D(q)=\lim _{a \rightarrow 0^{+}} D(q, a) / \ln a,
$$

which gives $D(h(q))$.

$D(h)$ is the fractal dimension of all points in the image having a local Holder exponent value of $h .^{20,21,23,27,48}$ A multifractal process has a heterogeneous spatial distribution of singularity strengths, which means that its roughness is assessed locally, and therefore has a corresponding singularity spectrum $D(h)$ with a concave down parabola shape, and the corresponding $\tau(q)$ spectrum is curved (non-linear). For a monofractal process, the spatial distribution of singularity strengths is homogeneous (i.e., the roughness is the same everywhere) and can be assessed globally. In this case, the singularity spectrum $D(h)$ collapses to a single point, where the global roughness is quantified via the Hurst exponent $H$, and the corresponding $\tau(q)$ spectrum is a linear function and is mathematically related to $H$ by $\tau(q)=q H-2 .^{20,21,23,27,48}$ An investigation of monofractal vs. multifractal processes therefore requires access to a range of $q$ values, i.e., $q \in\left(q_{\min }, q_{\max }\right)$, that is as large as possible. Generally speaking, the size of the image analyzed determines the range of $q$-values: the larger the image, the larger the range of $q$ values. $^{20,21,23,27,48}$

Clearly, there must be a balance between the size of the image and the risk of averaging out different monofractal signatures within the same image. It is that balancing effort that led to the choice of the size of the images in this study $(360 \times 360$ pixels, of which only the central $256 \times 256$ pixel part was kept for analysis to avoid edge effects). This allowed investigation of a range of $q$-values in the interval $q \sim$ -2 to $q \sim 3$, with more weight granted to $q$-values closer to 0 , as discussed below.

\section{C. Image sliding window analysis}

Each mammogram and its corresponding mask were fed to in-house 2D WTMM software, which consists of $\sim 130,000$ lines of $\mathrm{C}$ subroutines. A sliding $360 \times 360$-pixel window was used to divide mammograms into overlapping subregions, with 32-pixel shifts between subregion boundaries, running from left to right and top to bottom, starting at the top left corner of each image. For each subregion, the program first checked that the central $256 \times 256$-pixel section was entirely contained within the mask. If this condition was met, the program wavelet-transformed the subregion at 50 different size scales, from $a \sim 7$ pixels $(\sim 0.30 \mathrm{~mm})$ to $a \sim 120$ pixels $(\sim 5.0 \mathrm{~mm})$ and calculated the maxima chains (WTMM) and their WTMMM, the maxima lines, and the partition functions [Eq. (3)], $h(q, a)$ [Eq. (7)], and $D(q, a)$ [Eq. (8)]. After performing the WTMM calculations on each $360 \times 360$ subregion, only the central $256 \times 256$ pixels were considered for the WT skeleton to avoid edge effects. ${ }^{10,27,28}$

\section{D. Range of scales for multifractal analysis}

For each subregion that passed the mask test, a different sliding window technique was used to objectively determine the best range of scales to fit the power-law curves in the plots of $D(q, a)$ vs. $\log _{2}(a)$ (Fig. 1(f)) and $h(q, a)$ vs. $\log _{2}(a)$ (Figs. $1(\mathrm{~g})$ and $1(\mathrm{j}))$. The window varied along $\log _{2}(a)$ and was defined by a lower bound $\left(a_{\min }\right)$ and an upper bound $\left(a_{\max }\right)$ of $a$, which varied from $\log _{2}\left(a_{\min }\right)=0,0.1, \ldots, 2.1$ and from $\log _{2}\left(a_{\max }\right)=2.0,2.1, \ldots, 4.1$, respectively, in $\sigma_{w}$ units, where $\sigma_{w}=7$ pixels. All possible combinations of the lower and upper bounds, where the window was at least $\log _{2}\left(a_{\max }\right)-$ $\log _{2}\left(a_{\min }\right)=1.0$ wide, were considered. For each such $\left(a_{\min }\right.$, $\left.a_{\max }\right)$ window, $h(q=0)$ and $D(q=0)$ were calculated, as well as the goodness of fit $R^{2}$ of $h(q=0)$, denoted $R_{h(0)}^{2}$. The weighted standard deviation of $h$ over all values of $q$, denoted $s d_{w}$, and the weighted average of $R^{2}$ of $h(q, a)$ over all values of $q$, denoted $\left\langle R_{w}^{2}\right\rangle$, were also calculated, according to the weights listed in Table IV. For a $\left(a_{\min }, a_{\max }\right)$ window to be further considered, it had to satisfy all of the following conditions.

\section{D.1. $-0.2<h(q=0)<1.0$}

$h(q=0)$ is the Holder exponent, which represents the strength of the singularities of the image corresponding to the support dimension $D(q=0) .^{20,21,23,27,48}$ Although values outside of this range were not encountered in this study, obtaining very low values (high roughness) or high values (low roughness-note that a Hurst value of $H>1$ would mean that the underlying process is not only continuous, but differentiable) could be a flag for abnormally high noise levels or artificially smooth processes, respectively. 
TABLE IV. List of $q$ values and their associated weights.

\begin{tabular}{|c|c|c|c|c|c|c|c|c|c|c|c|c|c|c|c|c|c|}
\hline$q$ & 2 & 1.5 & 1 & 0.5 & 0.3 & 0.2 & 0.1 & 0 & 0.1 & 0.2 & 0.3 & 0.5 & 1 & 1.5 & 2 & 2.5 & 3 \\
\hline Weight & 0.1 & 0.5 & 1 & 3 & 5 & 7 & 9 & 10 & 9 & 8 & 7 & 5 & 3 & 2 & 1 & 0.5 & 0.2 \\
\hline
\end{tabular}

\section{D.2. $1.7<D(q=0)<2.5$}

$D(q=0)$ is the fractal dimension of the support of the image, also called the support dimension. ${ }^{20,21,23,27,48}$ Theoretically, for a rough surface representing an everywhere singular process, such as one would expect for the mammograms considered in this study, $D$ should be around 2.0. However, finite size effects may affect the manner in which the maxima lines multiply as the scale parameter $a$ approaches 0 .

\section{D.3. $R_{h(0)}^{2}>0.96$}

This condition ensured that the $h(q=0)$ curve was sufficiently linear to extract a reliable exponent from it.

\section{D.4. $s d_{w}<0.06$}

Requiring a low weighted standard deviation for $h$ ensured that subregions displaying multifractal scaling would be rejected, specifically when the slopes of the $h(q, a)$ curves were not within 0.06 of each other as $q$ varied. An example of a rejected subregion's $h(q, a)$ curves due to this condition not being satisfied is shown in Fig. 1(j) (black).

\section{D.5. $<R_{w}^{2}>>0.96$}

This condition ensured that all $h(q, a)$ curves were sufficiently linear, with more weight given to those curves closer to a value of $q=0$. An example of a rejected subregion's $h(q, a)$ curves due to this condition not being satisfied is shown in Fig. 1(j) (green).

If all possible $\left(a_{\min }, a_{\max }\right)$ windows of scales were rejected, the entire subregion was rejected (and displayed in gray as in Fig. 1(a)). If not, the subregions were classified via colors corresponding to the value of $h(q=0)$ (referred to simply as $H$ ) obtained from the $\left(a_{\min }, a_{\max }\right)$ window having the highest value of $R_{h(0)}^{2}$. After doing this for each subregion, the mammogram was returned as a small image of colored squares (Fig. 1(a)). Each square corresponded to one subregion of the original mammogram. Subregions where $H \leq 0.45$ (anti-correlated density fluctuations - fatty tissue) were colored blue, $0.45<H<0.55$ (uncorrelated density fluctuation - disrupted tissue) were yellow, and $H \geq 0.55$ (long-range correlated density fluctuation - dense tissue) were red. A sample case (DDSM, cancer 03, case 1022) is presented in Fig. 1(a), where the left (cancerous) and right MLO views were returned by the custom software.

\section{E. Left vs. right breast image registration}

To examine tissue differences between a patient's tumorous breast vs. opposite (healthy) breast, the tumorous breast was aligned with its opposite using a thin-plate spline, ${ }^{49}$ post sliding window WTMM analysis (Fig. 2). In the normal population, a breast was chosen at random (left or right) and aligned to the opposite breast. The thin-plate spline tools in the Momocs R library ${ }^{50}$ were used to predict a shift in coordinate positions from points in the breast undergoing registration to the opposite breast. These shifts were added to the breast undergoing registration. A resulting point in the registered breast that matched a coordinate of a point in the opposite breast was called a corresponding point. Corresponding points in registered breasts were compared to opposite breasts for color changes (blue, red, yellow). The total number of squares that changed color were called the number of squares transitioned (see section 2.F. below).

\section{F. Metrics}

To explore potential differences in spatial organization of the mammogram roughness of different subregions (either blue, $H<=0.45$; yellow $0.45<H<0.55$; red, $H>=0.55$ ), the following metrics were used: (a) the number of yellow squares (subregions) per breast (NY), (b) the number of clusters of yellow squares (allowing horizontal, vertical, and diagonal connections between squares) (NCY), (c) the number of squares transitioned (NT), and (d) a combination score

$$
\Xi(\alpha)=\frac{(\mathrm{NY}) *(\mathrm{NCY}) *(\mathrm{NT})}{\left(\sum \text { All Squares }\right)^{\alpha}},
$$

where $\alpha$ is an exploratory exponent that was allowed to vary from 0 to 4.0. A continuous density fluctuation score

$$
\rho=\frac{\left(\sum \text { Red Squares }\right)}{\left(\sum \text { Red Squares }\right)+(\gamma)\left(\sum \text { Blue Squares }\right)},
$$

where $\gamma$ was an adjustment factor, was used to objectively describe the density of breasts. This was compared to the subjective Breast Imaging Reporting and Data System (BIRADS) scoring of breast density by radiologists: (a) fatty, (b) scattered areas of fibroglandular density, (c) heterogeneously dense, (d) extremely dense, which are subjective and based on individual radiologist experience. The numerical determination of $\alpha$ and $\gamma$ is discussed below.

\section{G. Data mining}

A data mining approach was used to maximize quantifiable observed differences between normal and cancer cases.

Each mammogram contained rejected subregions (see section 2.C.). If the percent of rejected subregions in the mammogram was too high, the image was removed from the analysis. To determine this empirically and objectively, the 

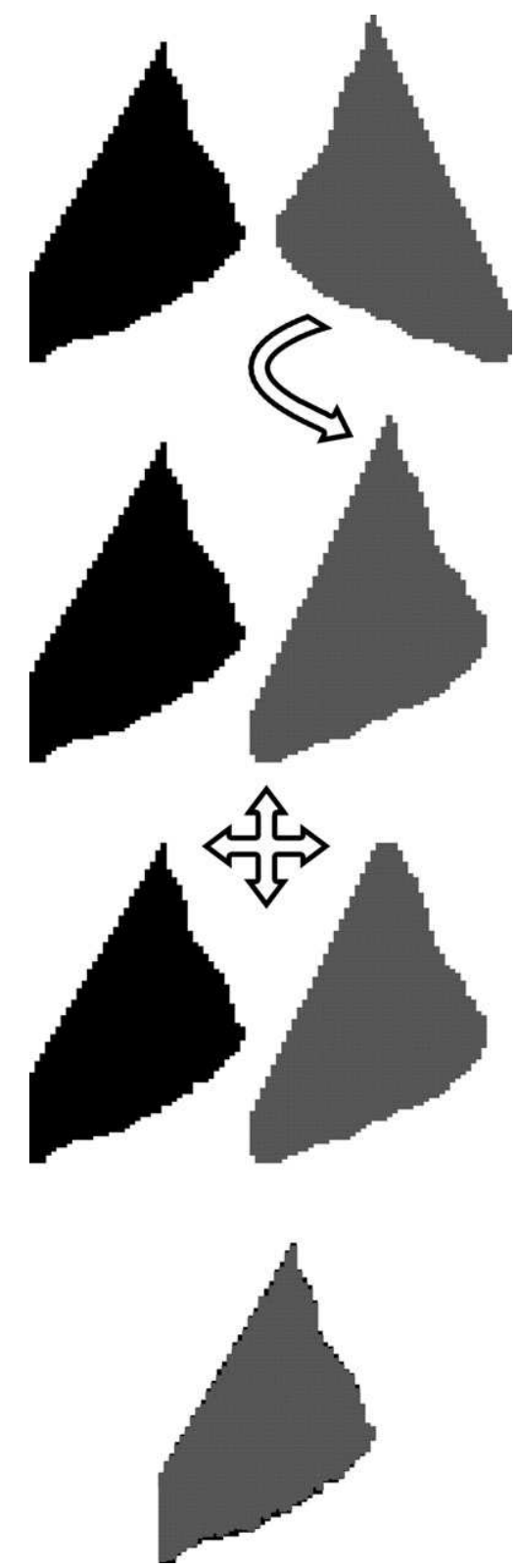

FIG. 2. Image registration of a breast to the opposite breast. Same case as shown in Fig. 1 (DDSM cancer 03, case 1022), with the left breast shown on right (dark gray) and the right breast shown on left (light gray). The left breast (dark gray) is first flipped horizontally, and then aligned to the oppo site breast using a thin plate spline algorithm. The bottom panel shows an overlay of the registered breasts.

maximum allowable percent of rejected subregions was varied between 0 and 40 percent in increments of 1 percent. Similarly, the minimum number of connected yellow squares needed to qualify as a yellow cluster was varied between 5 and 200 in increments of 5. The $\alpha$ parameter for $\Xi(\alpha)$ [Eq. (11)] was varied between 0 and 4 in increments of 0.5 . To calculate the $\gamma$ that yielded $\rho$ [Eq. (12)] scores that best correlated with BIRADS scores, $\gamma$ was varied from 0 to 1 in increments of 0.01 . For every possible combination of values of the above parameters, the statistical significances of differences in normal and cancer cases given by each metric were calculated. The combination that yielded the greatest statistical significance for the greatest number of metrics was selected. Using this approach, the objectively determined allowable percent of rejected subregions was 30 percent. A yellow cluster was defined as having 45 or more connected yellow subregions. An $\alpha$ of 1.5 [Eq. (11)] and a $\gamma$ of 0.82 [Eq. (12)] were selected.

After these parameters were objectively determined via the data mining approach on the normal vs. cancer cases, the benign cases were analyzed (normal vs. benign and benign vs. cancer) using the parameters unaltered.

\section{H. Statistical analysis}

All statistical distribution analyses, significance tests, density distribution, cumulative density, Wilcoxon tests, and goodness of fit, were performed using the R language.

\section{RESULTS}

\section{A. Quantitating loss of tissue homeostasis in tumorous breasts}

All metrics displayed statistically significant differences between normal and tumorous (cancer or benign) cases (Fig. 3). Tumorous breasts had a higher number of yellow (disrupted) squares than normal breasts $(P \sim 0.0423$ for cancer, $P \sim 0.0009$ for benign, Figs. 3(a) and 3(b)), supporting the hypothesis that tumor cases had greater loss of tissue homeostasis than normal cases. The same was true for the number of yellow clusters $(P \sim 0.0032$ for cancer, $P \sim 0.0077$ for benign, Fig. 3(c) and 3(d)), suggesting that tumor-affected microenvironment may be identified by yellow clusters. Tumor cases also had a higher number of transitioned squares than normal cases $(P \sim 0.0346$ for cancer, $P \sim 0.0009$ for benign, Fig. 3(e) and 3(f)), indicating that tumor cases exhibited greater asymmetry between tumorous and opposite breasts than normal cases exhibited between two healthy breasts. Combining these relationships into $\Xi(1.5)$ yielded that both cancer and benign cases were distinguishable from normal cases via this metric $(P \sim 0.0023$ for cancer, $P \sim$ 0.0049 for benign, Fig. 3(g) and 3(h)). Finally, when grouping the tumorous cases together (cancer and benign) and comparing them as a unit to normal cases, the significance level was even greater $(P \sim 0.0006)$.

\section{B. Tissue disruption and change in tissue density vs. age}

In the cancer population, the number and percent of yellow squares, which represent disrupted tissue, did not vary significantly with age (Figs. 4(a) and 4(b), stars). However, in the normal and benign populations, the number of yellow squares increased with age $[P \sim 0.0376$ for normal, Fig. 4(a), black dots; $P \sim 0.0309$ for benign, Fig. 4(a), triangles], but not the percent of yellow squares [Fig. 4(b), black dots and triangles].

The number and percent of blue squares, representing fatty tissue, increased with age in the cancer population $[P \sim 0.0029$, 

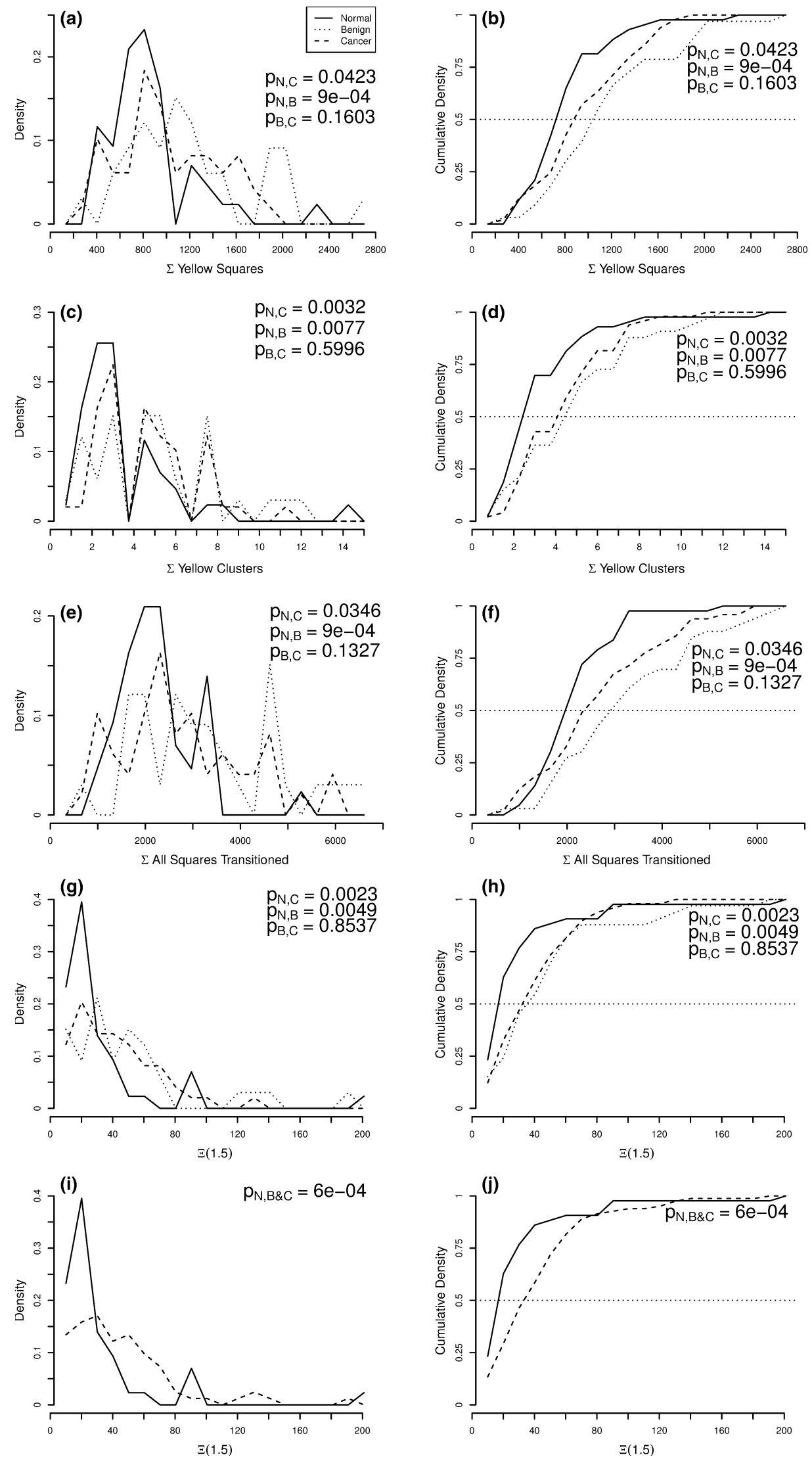

Fig. 3. Density distribution and cumulative density of metrics in normal, cancer, and benign populations. (a), (b) Distribution of total number of yellow squares. (c), (d) Distribution of total number of yellow clusters. (e), (f) Distribution of total number of squares transitioned. (g), (h) Distribution of the combination metric $\Xi(1.5)$ [Eq. (11)]. (i), (j) Distribution of the combination metric for normal vs. tumor (benign and cancer combined). 

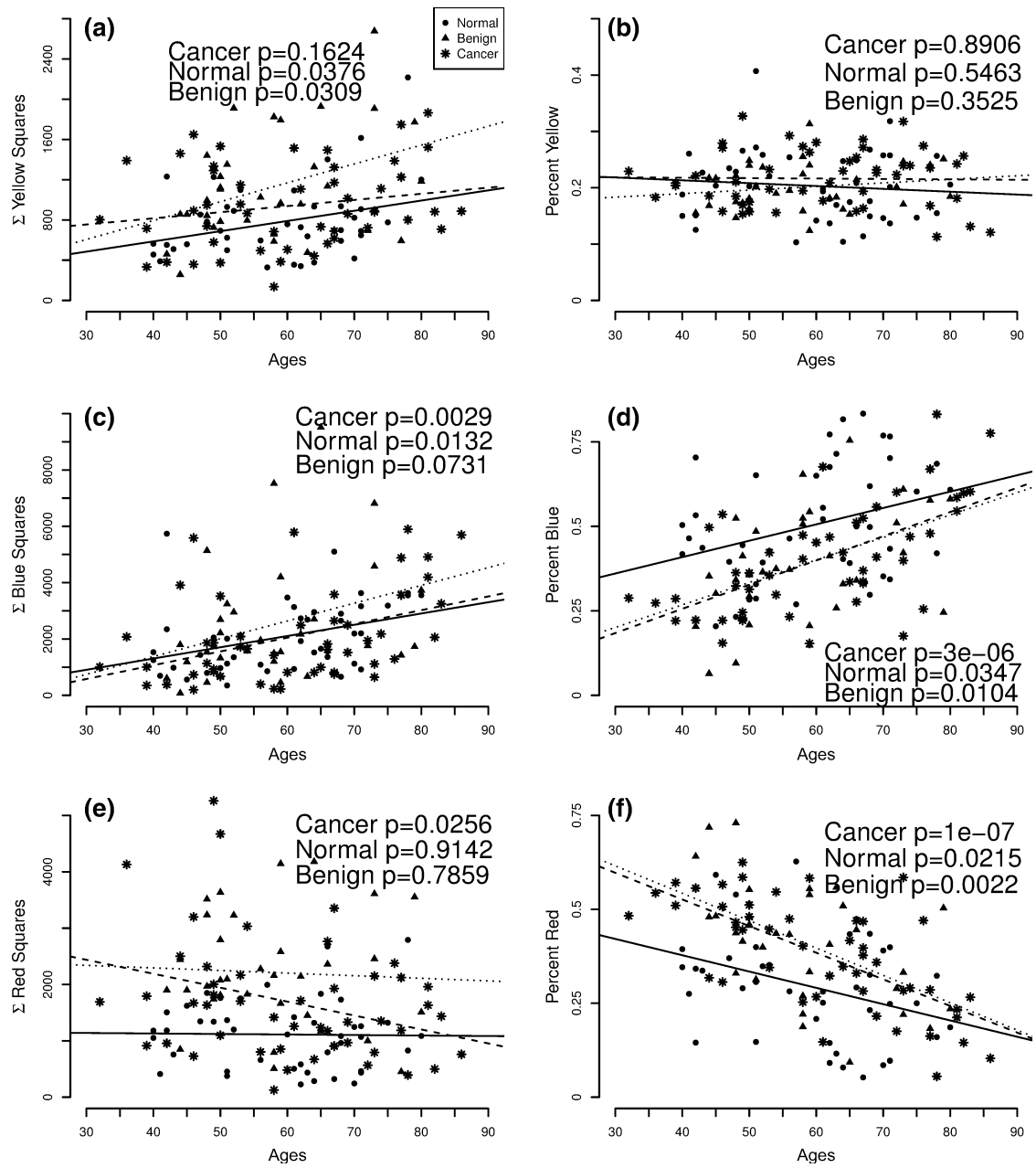

FIG. 4. Metrics distinguishing tissue types. The left hand column (a), (c), (e) displays number of yellow, blue, and red squares, respectively, vs. age. The right hand column (b), (d), (f), displays percent of yellow, blue, and red squares, respectively, vs. age. Red squares represent dense tissue, blue squares represent fatty tissue, and yellow squares represent uncorrelated (disrupted) tissue. Linear fits are shown in full, dashed, and dotted lines for normal, cancer, and benign popula tions, respectively.

Fig. 4(c), stars, and $P \sim 3 \mathrm{E}-6$, Fig. 4(d), stars, respectively] as well as in the normal population $[P \sim 0.0132$, Fig. $4(\mathrm{c})$, black dots, and $P \sim 0.0347$, Fig. 4(d), black dots, respectively]. For the benign cases, the significance was only achieved in the percent of blue squares $[P \sim 0.0104$, Fig. $4(\mathrm{~d})$, triangles], but not in the number of blue squares [Fig. 4(c), triangles].

The number and percent of red squares, representing dense tissue, decreased with age in the cancer population $[P$ $\sim 0.0256$, Fig. 4(e), stars, and $P \sim 1 \mathrm{E}-7$, Fig. 4(f), stars, respectively], while in the normal and benign populations, the number of red squares did not vary as a function of age (Fig. 4(e), black dots and triangles, respectively), but the percent of red squares decreased with age for both normal $[P \sim$ 0.0215 , Fig. 4(f), black dots) and benign cases $(P \sim 0.0022$, Fig. 4(f), triangles].

\section{C. Toward a continuous density fluctuation score}

A continuous density fluctuation score [Eq. (12) and Fig. 5] was proposed to numerically and objectively score the density of breasts and could eventually be considered an accompaniment to subjective BIRADS scores. While the continuous density fluctuation score was successfully able to distinguish between most BIRADS density scores $(P<0.001$ or better), it could not distinguish density 1 breasts from density 2 breasts, nor density 3 breasts from density 4 breasts.

\section{DISCUSSION}

For scale-invariant rough surfaces such as mammograms, a Fourier-based power spectrum analysis (or related technique) yields a slope of $\beta=\tau(2)+4{ }^{20,21,23,27,48}$ Clearly, by only investigating one specific value of $q=2$, it is mathematically impossible for a power spectral analysis to discriminate between monofractal (linear $\tau(q)$ ) vs. multifractal (nonlinear $\tau(q)$ ) rough surfaces. This is a key argument, confirming the need for a multifractal formalism to investigate mammographic breast tissue: some subregions may display multifractal scaling. However, these are likely false multifractal signatures created by a juxtaposition of two or more regions within a single image, each having their own monofractal signatures. These regions were rejected from the analysis (see 


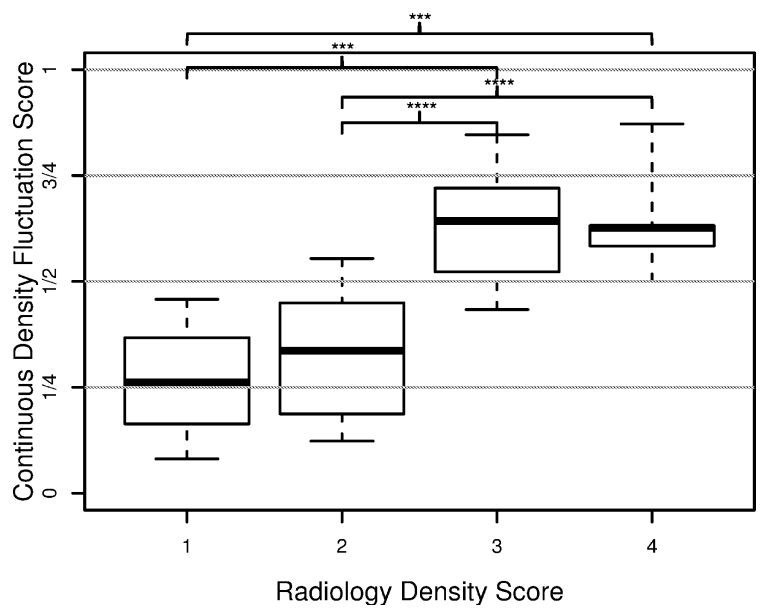

FIG. 5. Continuous density fluctuation score [Eq. (12)] vs. radiological den sity score for classification of breast tissue density of normal cases. Statistical significance level is indicated by the horizontal brackets $(* * *$ for $P<0.001$; **** for $P<0.0001)$.

Fig. 1(j)). Again, it is important to emphasize that a power spectral analysis would not have been able to correctly categorize these regions potentially composed of fatty tissue $(H<1 / 2)$ and dense tissue $(H>1 / 2)$, and could have erroneously reported an uncorrelated process $(H \sim 1 / 2)$.

The relationships between metrics and age suggest the general trend that dense tissue (red squares) decreases with age while fatty tissue (blue squares) increases with age. This is in line with the established relationship of mammographic density and age. ${ }^{51}$ Another observable trend is that disrupted tissue (yellow squares) stays relatively constant with age in cancerous patients, but increases in normal and benign patients (albeit for the number of squares only, not for the percent). If this observation is further validated, it would support the hypothesis that loss of tissue homeostasis and cancer risk increase in likelihood with age. ${ }^{1,2}$

It is worth noting there were significantly fewer density 1 cases than density 2 cases (4 vs. 17 cases, respectively) in the normal population data, which likely affected statistics for the continuous density fluctuation score. Applying the continuous density fluctuation score to a larger population may improve the correlations found with the radiologist BIRADS score.

\section{CONCLUSION}

Differences in tumorous and normal breast cases were observable and quantifiable using the objective sliding window WTMM analysis of entire breast mammograms developed and used in this paper. This was done on scanned mammographic film from the (often imperfect) DDSM data. This proof of concept suggests future work using high-quality full field digital mammography (FFDM).

Several sets of parameters introduced in this paper are preliminary and require future refinement. For example, the definition of the yellow range, $(0.45<H<0.55)$, the weights that were chosen for each $q$ value, the step size for the image sliding window approach (32 pixels), etc., should all be revisited, with FFDM. The fully objective and automated approach to determine the choice of minimum and maximum scales to extract critical exponents was introduced here for the first time using the 2D WTMM method.

The metrics presented here are also objective and are likely to exhibit quantifiable changes over the lifetime of a patient, based on identified correlations with age. Given this, it may be possible to observe tissue disruption early and use it to pre-identify potential danger zones. Future work should not only employ FFDMs, but should be longitudinal. This will enable examination of changes in these (and new) metrics over time in greater detail and ultimately on a patient-bypatient basis (rather than in population studies like this one). Indeed, in the breast, signs of aberrant stroma and epithelia may exist long before there is overt carcinoma. ${ }^{1-3,52}$ In fact, it has already been suggested that tissue disruption might not always be reactive, but might sometimes play an initial role in breast carcinogenesis. ${ }^{1-3,52}$ This motivates the development of new metrics such as the ones presented herein that could detect cancer at an earlier stage and help better understand the biophysics of loss of tissue homeostasis.

\section{ACKNOWLEDGMENTS}

The authors wish to thank the Maine Cancer Foundation for their grant and their continuing support of this research. Thanks to Veronique Maguer-Satta for beneficial discussions. Also thanks to CompuMAINE Lab members Shawn Firouzian, Hannah Dewey, Seth Albert, Elliot Ossanna, and Derrick Cox for technical discussions.

\section{CONFLICTS OF INTEREST}

We declare the patent application: PCT/US14/35153.

Title: IMPROVED METHODS OF CANCER DETECTION.

Applicant: University of Maine System Board of Trustees.

Filed: April 23, 2014 Inventors: Andre Khalil \& Kendra Batchelder.

\footnotetext{
a) Author to whom correspondence should be addressed. Electronic mail: andre.khali1@maine.edu.
}

\section{REFERENCES}

1. Bissell MJ, Hines WC. Why don't we get more cancer? A proposed role of the microenvironment in restraining cancer progression. Nat Med. 2011:17:320 329 .

2. Maguer Satta V. The stem cell niche: the black master of cancer. In: Shostak S, eds. Cancer Stem Cells Theories and Practice. Rijeka: InTech; 2011.

3. Alowami S, Troup S, Al Haddad S, Kirkpatrick I, Watson PH. Mammo graphic density is related to stroma and stromal proteoglycan expres sion. Breast Cancer Res. 2003;5:R129 R135.

4. Van der Auwera I, Bovie C, Svensson C, et al. Quantitative methylation profiling in tumor and matched morphologically normal tissues from breast cancer patients. BMC Cancer. 2010;10:97. 
5. Yan PS, Venkataramu C, Ibrahim A, et al. Mapping geographic zones of cancer risk with epigenetic biomarkers in normal breast tissue. Clin Cancer Res. 2006;12:6626 6636.

6. Tanner K, Mori H, Mroue R, Bruni Cardoso A, Bissell MJ. Coherent angular motion in the establishment of multicellular architecture of glan dular tissues. Proc Natl Acad Sci U S A. 2012;109:1973 1978.

7. Dromain C, Boyer B, Ferre R, Canale S, Delaloge S, Balleyguier C. Computed aided diagnosis (CAD) in the detection of breast cancer. Eur J Radiol. 2013;82:417 423.

8. Ganesan K, Acharya UR, Chua CK, Min LC, Abraham KT, Ng KH. Computer aided breast cancer detection using mammograms: a review. IEEE Rev Biomed Eng. 2013;6:77 98.

9. Jalalian A, Mashohor SB, Mahmud HR, Saripan MI, Ramli AR, Karasfi B. Computer aided detection/diagnosis of breast cancer in mammogra phy and ultrasound: a review. Clin Imaging. 2013;37:420 426.

10. Batchelder KA, Tanenbaum AB, Albert S, et al. Wavelet based 3d reconstruction of microcalcification clusters from two mammographic views: new evidence that fractal tumors are malignant and euclidean tumors are benign. PLoS One. 2014;9:e107580.

11. Plourde SM, Marin Z, Smith ZR, Toner BC, Batchelder KA, Khalil A. Computational growth model of breast microcalcification clusters in simulated mammographic environments. Comput Biol Med. 2016;76:7 13.

12. Huo Z, Giger ML, Olopade OI, et al. Computerized analysis of digitized mammograms of BRCA1 and BRCA2 gene mutation carriers. Radiol. 2002;225:519 526.

13. Manduca A, Carston MJ, Heine JJ, et al. Texture features from mammo graphic images and risk of breast cancer. Cancer Epidemiol Biomarkers Prev. 2009;18:837 845.

14. Nicolis O, R. C. P, Vidakovic B. 2D wavelet based spectra with applica tions. Comput Stat Data An. 2011;55:738 751.

15. Ramirez Cobo P, V. B. 2D wavelet based multiscale approach with applications to the analysis of digital mammograms. Comput Stat Data An. 2013;58:71 81.

16. Li H, Giger ML, Olopade OI, Chinander MR. Power spectral analysis of mammographic parenchymal patterns for breast cancer risk assessment. J Digit Imaging. 2008;21:145 152.

17. Gierach GL, Li H, Loud JT, et al. Relationships between computer extracted mammographic texture pattern features and BRCA1/2 mutation status: a cross sectional study. Breast Cancer Res. 2014;16:424.

18. Heine JJ, Velthuizen RP. Spectral analysis of full field digital mammog raphy data. Med Phys. 2002;29:647 661.

19. Muzy JF, Bacry E, Arneodo A. Wavelets and multifractal formalism for singular signals: application to turbulence data. Phys Rev Lett. 1991;67:3515 3518.

20. Muzy JF, Bacry E, Arneodo A. Multifractal formalism for fractal sig nals: the structure function approach versus the wavelet transform mod ulus maxima method. Phys Rev E Stat Phys Plasmas Fluids Relat Interdiscip Topics. 1993;47:875 884.

21. Muzy JF, Bacry E, Arneodo A. The multifractal formalism revisitied with wavelets. Int J Bifurc Chaos. 1994;4:245 302.

22. Arneodo A, Bacry E, Muzy JF. The thermodynamics of fractals revisited with wavelets. Phys A. 1995;213:232 275.

23. Arneodo A, Decoster N, Roux S. A wavelet based method for multifrac tal image analysis. I. Methodology and test applications on isotropic and anisotropic random rough surfaces. Eur Phys J B. 2000;15:567 600 .

24. Decoster N, Arneodo A, Roux S. A wavelet based method for multifrac tal image analysis. II. Applications to synthetic multifractal rough sur faces. Eur Phys J B. 2000;15:739 764 .

25. Roux S, Decoster N, Arneodo A. A wavelet based method for multifrac tal image analysis. III. Applications to high resolution satellite images of cloud structure. Eur Phys J B. 2000;15:765 786.

26. Kestener P, Lina JM, St Jean P, Arneodo A. Wavelet based multifractal formalism to assist in diagnosis in digitized mammograms. Image Anal Stereol. 2001;20:169 174.

27. Khalil A, Joncas G, Nekka F, Kestener P, Arneodo A. Morphological analysis of HI features. II. Wavelet based multifractal formalism. Astro phys J Suppl S. 2006;165:512 550.

28. Khalil A, Aponte C, Zhang R, et al. Image analysis of soft tissue in growth and attachment into highly porous alumina ceramic foam metals. Med Eng Phys. 2009;31:775 783.
29. Gerasimova E, Audit B, Roux SG, et al. Multifractal analysis of dynamic infrared imaging of breast cancer. EPL. 2013;104:68001.

30. Gerasimova E, Audit B, Roux SG, et al. Wavelet based multifractal analysis of dynamic infrared thermograms to assist in early breast cancer diagnosis. Front Physiol. 2014;5:176.

31. Caddle LB, Grant JL, Szatkiewicz J, et al. Chromosome neighborhood composition determines translocation outcomes after exposure to high dose radiation in primary cells. Chromosome Res. 2007;15:1061 1073.

32. Khalil A, Grant JL, Caddle LB, Atzema E, Mills KD, Arneodo A. Chro mosome territories have a highly nonspherical morphology and nonran dom positioning. Chromosome Res. 2007;15:899 916.

33. Snow CJ, Goody M, Kelly MW, et al. Time lapse analysis and mathe matical characterization elucidate novel mechanisms underlying muscle morphogenesis. PLoS Genet. 2008;4:e1000219.

34. Snow CJ, Peterson MT, Khalil A, Henry CA. Muscle development is disrupted in zebrafish embryos deficient for fibronectin. Dev Dyn. 2008;237:2542 2553.

35. Grant J, Verrill C, Coustham V, et al. Perinuclear distribution of hete rochromatin in developing C. elegans embryos. Chromosome Res. 2010;18:873 885 .

36. Goody MF, Kelly MW, Lessard KN, Khalil A, Henry CA. Nrk2b mediated NAD+ production regulates cell adhesion and is required for muscle morphogenesis in vivo: Nrk2b and NAD+ in muscle morpho genesis. Dev Biol. 2010;344:809 826.

37. Kestener P, Arneodo A. Three dimensional wavelet based multifractal method: the need for revisiting the multifractal description of turbulence dissipation data. Phys Rev Lett. 2003;91:194501.

38. Kestener P, Arneodo A. Generalizing the wavelet based multifractal for malism to vector valued random fields: application to turbulent velocity and vorticity 3D numerical data. Phys Rev Lett. 2004;93:044501.

39. Boyd NF, Lockwood GA, Byng JW, Tritchler DL, Yaffe MJ. Mammo graphic densities and breast cancer risk. Cancer Epidemiol Biomarkers Prev. 1998;7:1133 1144.

40. Santen RJ, Yue W, Heitjan DF. Occult breast tumor reservoir: biological properties and clinical significance. Horm Cancer. 2013;4:195 207.

41. Nielsen M, Thomsen JL, Primdahl S, Dyreborg U, Andersen JA. Breast cancer and atypia among young and middle aged women: a study of 110 medicolegal autopsies. Br J Cancer. 1987;56:814 819.

42. Heath M, Bowyer K, Kopans D, et al. Current status of the digital database for screening mammography. In: Fourth International Workshop on Digi tal Mammography. Norwell, MA: Kluwer Academic Publishers; 1998.

43. Heath M, Bowyer K, Kopans D, Moore R, Kegelmeyer WP. The digital database for screening mammography. In: Fifth International Workshop on Digital Mammography. Madison, WI: Medical Physics Publishing; 2001.

44. Goody MF, Kelly MW, Reynolds CJ, Khalil A, Crawford BD, Henry CA. Nad+ biosynthesis ameliorates a zebrafish model of muscular dys trophy. PLoS Biol. 2012;10:e1001409.

45. Kestener P, Conlon PA, Khalil A, et al. Characterizing complexity in solar magnetogram data using a wavelet based segmentation method. Astrophys J. 2010;717:995 1005.

46. McAteer RTJ, Kestener P, Arneodo A, Khalil A. Automated detection of coronal loops using a wavelet transform modulus maxima method. Sol Phys. 2010;262:387 397.

47. Roland T, Khalil A, Tanenbaum A, et al. Revisiting the physical pro cesses of vapodeposited thin gold films on chemically modified glass by atomic force and surface plasmon microscopies. Surf Sci. 2009;603:3307 3320 .

48. Arneodo A, Decoster N, Kestener P, Roux SG. A wavelet based method for multifractal image analysis: from theoretical concepts to experimen tal applications. Adv Imaging Electr Phys. 2003;126:1 92.

49. Green PJ, Silverman BW. Nonparametric Regression and General ized Linear Models: A Roughness Penalty Approach. Monographs on Statistics and Applied Probability 58. Boca Raton, FL: Chapman \& Hall; 1993.

50. Bonhomme V, Picq S, Gaucherel C, Claude J. Momocs: outline analysis using R. J Stat Softw. 2014;56:24.

51. Checka CM, Chun JE, Schnabel FR, Lee J, Toth H. The relationship of mammographic density and age: implications for breast cancer screen ing. AJR Am J Roentgenol. 2012;198:W292 W295.

52. Kinzler KW, Vogelstein B. Landscaping the cancer terrain. Science. 1998;280:1036 1037. 